\title{
A Pre-Experimental Study to Assess the Effect of Psycho-social Nursing Intervention on Happiness and Psychological Well Being among Adolescents in Selected Schools of Amritsar, Punjab
}

\author{
Rimplepreet Kaur ${ }^{1}$, Sukhbir Kaur ${ }^{2}$, Gurjeet Kaur ${ }^{3}$ \\ ${ }^{1}$ M.Sc. (N) Student, SGRD College of Nursing, Vallah, Amritsar \\ ${ }^{2}$ Associate Professor, SGRD College of Nursing, Vallah, Amritsar \\ ${ }^{3}$ Assistant Professor, SGRD College of Nursing, Amritsar
}

Corresponding Author: Rimplepreet Kaur

\begin{abstract}
Adolescents are tomorrow's adult population, and their health and well being are crucial. Over the past few decades, adolescents are increasingly experiencing mental health challenges. As in schools, adolescent's happiness and psychological well being has become a more important topic among their parents and teachers. The objective of the study was to assess the effect of psycho-social nursing intervention on happiness and psychological well being among adolescents at selected schools of Amritsar. A Pre experimental study (one group pretest and post test) was conducted on 60 adolescents studying in public schools of Amritsar selected by purposive sampling technique. The adolescents were allocated into three groups of 20 each. 12 sessions ( 5 sessions / week) of psycho-social nursing intervention were given to each group for 1 hour duration. Data was collected by administering standardized happiness and psychological well being questionnaire. The result of the study revealed that the mean post-test happiness score was higher $(84.83 \pm 12.12)$ than pre test mean score of happiness $(50.70 \pm 8.67)$ and was found to be statistically significant $\left(\mathrm{t}=20.30, \mathrm{df}=59, \mathrm{p}=0.000^{* *}\right)$. The mean post-test psychological well being score was higher $(174.63 \pm 28.01)$ than pre-test mean score of psychological well being (97.18 \pm 22.71$)$ and was found to be statistically significant ( $t=20.61, \mathrm{df}=59$, $\left.\mathrm{p}=0.001^{*}\right)$ but there was no significant association was found between pretest and post-test score of level of happiness and psychological well being with selected demographic variables. The study concluded that psycho-social nursing intervention was effective to improve the level of happiness and psychological well being among adolescents studying at selected public schools of Amritsar.
\end{abstract}

Key words: psycho-social nursing intervention, happiness, psychological well being, adolescents, school

\section{INTRODUCTION}

Adolescents are tomorrow's adult population or future generation, and their health and well being are crucial and pivotal. Thus, the interest in the health of adolescents is something new, and focuses on their happiness and well being even more so. ${ }^{1}$

As adolescent girls and boys grow with their age, they take on additional or extra responsibilities, experiment with new ways of doing things and press for selfdetermination and independence. It is times in which values and skills are developed that have great impact on adolescent's happiness and psychological well-being. ${ }^{2}$

Over the past few decades, adolescents are increasingly experiencing mental health challenges. Research studies shows that 1 in 10 children aged 13-18 
currently having, or will develop a serious mental condition. ${ }^{3}$

Now-a-days, there are many mental health problems which may develop or first become apparent during adolescence such as mood disorders, anxiety disorders, and thought disorders as well as psychosocial disorders. Suicide is a major reason of death for this age group. Eating disorders, such as anorexia nervosa and bulimia nervosa, are more in girls and most commonly develop during adolescence. ${ }^{4}$

Happiness can be understood as an outcome of life and has a major influence on positive psychological well being. (Veenhoven 2010). ${ }^{5}$ According to Ryan and Deci, (2001) Psychological well-being is defined as full engagement and optimal performance in the existential challenges of life. $^{6}$

As in schools, adolescent's happiness and psychological well being has become a more important topic among their parents and teachers. They develop plans for students to achieve academic success; they set high expectations and may become anxious about their academic performance and ability to reach their desired goals. They cope with issues through sustained performance, and maintaining a sense of well-being as they propel themselves toward their goals. ${ }^{7}$

Psycho-social nursing intervention refers to the set of exercises which are designed to enhance happiness and psychological well being among adolescents. The main concept of psychosocial nursing intervention is to ventilate the feelings and to boost self-esteem and selfconfidence along with the good psychological well being and competent level of happiness through various alternatives or exercises like take a daily vacation, self compassion letter, three minute breathing space, write your future diary, three funny things, the best possible self, forgiveness interventions, meditative intervention etc. $^{8}$

The major goal of the study is to increase students' ability to handle day to- day stressors and problems that are common for most students during adolescence. It promotes optimism by teaching students to think more realistically and flexibly about the problems they encounter. It also teaches assertiveness, creative brainstorming, decision making, relaxation, and several other coping and problem solving skills. It may also help to lower the cases of depression in adolescents and also decreases the suicidal risks. ${ }^{9}$

\section{RESEARCH PROBLEM}

A Pre-Experimental Study to Assess the Effect of Psycho-social Nursing Intervention on Happiness and Psychological Well Being among Adolescents in Selected Schools of Amritsar, Punjab.

\section{OBJECTIVE}

1. To assess the pre-intervention happiness and psychological well being among adolescents.

2. To assess the effect of psycho-social nursing intervention on happiness and psychological well being among adolescents.

3. To find out the relationship between happiness and psychological well being among adolescents.

4. To find out the association of happiness and psychological well being among adolescents with selected socio demographic variables.

\section{OPERATIONAL DEFINITION}

EFFECT: refers to impact of psycho-social nursing interventions on adolescents.

PSYCHO-SOCIAL

NURSING

INTERVENTION: refers to the set of activities and exercises designed to enhance happiness and psychological well-being of adolescents.

HAPPINESS: refers to the adolescents who feel good or positive at schools as measured by standardized questionnaire for happiness.

PSYCHOLOGICAL WELL BEING: refers to the state in which an adolescent feels psychological fit or well with his 
surroundings measured by standardized questionnaire for psychological well-being.

ADOLESCENTS: the students between the age of 14-16 years who are studying in 8th, 9th and 10th standard.

SCHOOLS: refers to the public schools of Amritsar that are providing education from 1st to 12th classes and affiliated to Central Board of Secondary Education (CBSE Board).

\section{MATERIALS AND METHODS}

RESEARCH DESIGN: The present study was Pre - experimental, one group pre-test post-test design was used.

RESEARCH SETTING: The present study was conducted at Shri HarKrishan Sen. Sec. school, Amritsar and Khalsa Sen. Sec. school, Amritsar.

TARGET POPULATION: Target population for present study includes 60 adolescents studying at Shri Guru Harkrsihan Public Sen. Sec. School Amritsar and Khalsa Sen. Sec. School, Amritsar.

SAMPLING TECHNIQUE: Nonrandomized Purposive sampling technique was used to select the sample for the study.

SAMPLE SIZE: The sample for the present study comprises of 60 adolescents who scored average and below average in happiness and moderate and below moderate psychological well being studying in public schools of Amritsar, Punjab.

\section{VARIABLES UNDER STUDY:}

Independent variables: Psycho-Social Nursing Intervention

Dependent variables: Happiness and Psychological Well being among adolescents.

\section{INCLUSION CRITERIA}

Adolescents' who are:-

- Having average and below average happiness and moderate and below moderate psychological well being.

- Present during the time of data collection.

- Willing to participate.
- Between the age of 14-16 years.

- Studying in 8th, 9th and 10th standard.

\section{EXCLUSION CRITERIA:}

Adolescents who are:-

- Having above average happiness and psychological well being.

- Physical or mentally ill.

- Exercising other techniques for happiness and well being like attending Yoga classes etc.

\section{DESCRIPTION OF TOOLS}

\section{PART-A:- Socio- Demographic variables}

It includes items for obtaining personal information's of participants i.e. age, gender, religion, habitat, class, type of family, father's education, mother's education, father's occupation, mother's occupation, entertainment facilities used, pocket money used on.

PART- B:- Standardized happiness scale by Dr. R.L.Bhardwaj and Dr. Poonam R. Das was used to assess the level of happiness among adolescents and consist of 28 questions.

Scoring: Total items in the questionnaire are 28. It is a five-point Likert scale. The scoring for questions were $1,2,3,4,5$ respectively. Reverse scoring for 4 questions (question no. 6, 9, 10, 14).

\section{SCORE INTERPRETATION FOR HAPPINESS SCALE:}

Unhappiness (upto 20), Less happiness (2030), Average (31-60), High happiness (6179), Greater happiness (80+).

PART-C:- Standardized tool Psychological well being by Dr. Davendra Singh Sisodia and Ms. Pooja Choudary was used to assess the level of psychological well being among adolescents which consisted of 50 items divided across five domains i.e. Satisfaction, Efficiency, Sociability, Mental Health and Interpersonal relationship. being:

Domains of Psychological well

- Satisfaction: question under this domain 1 to 10 . 
- Efficiency: question under this domain 11 to 20

- Sociability: question under this domain 21 to 30

- Mental health: question under this domain 31 to 40

- Interpersonal relationship: question under this domain 41 to 50

\section{SCORE INTERPRETATION FOR} PSYCHOLOGICAL WELL BEING:

Strongly agree (5), Agree (4), Undecided (3), Disagree (2), Strongly disagree (1)

\section{NORMS FOR INTERPRETATION OF THE RAW SCORES (FOR EACH AREA/ DOMAIN WISE)}

Very low (10-12), Low (12-16), Moderate (16-43), High (43-48), Very high (48-50)

\section{NORMS FOR INTERPRETATION FOR THE RAW SCORES (FOR ENTIRE SCALE)}

Very Low (50-58), Low (58-83), Moderate (83-217), High (217-242), Very high (242250)

Table 2 depicts that in post-test $21.7 \%$ were having average happiness and less happiness whereas in pre-test all were having (100\%) unhappiness level.
Table 1: Frequency and percentage distribution of adolescents according to demographic variables, $\mathbf{N}=\mathbf{6 0}$

\begin{tabular}{|c|c|c|c|}
\hline S.No & Demographic Variable & $\begin{array}{l}\text { Frequency } \\
\text { (f) }\end{array}$ & $\begin{array}{l}\text { Percentage } \\
\text { (\%) }\end{array}$ \\
\hline 1. & $\begin{array}{l}\text { Age (in Years) } \\
\text { a. } \quad 14 \mathrm{yrs} \\
\text { b. } 15 \mathrm{yrs} \\
\text { c. } \quad 16 \mathrm{yrs}\end{array}$ & $\begin{array}{l}19 \\
20 \\
21\end{array}$ & $\begin{array}{l}31.7 \\
33.3 \\
35\end{array}$ \\
\hline 2 & \begin{tabular}{l}
\multicolumn{2}{l}{ Gender } \\
a. $\quad$ Male \\
b. Female
\end{tabular} & $\begin{array}{l}30 \\
30\end{array}$ & $\begin{array}{l}50 \\
50\end{array}$ \\
\hline 3 & \begin{tabular}{ll}
\multicolumn{2}{l}{ Religion } \\
a. & Sikh \\
b. & Hindu \\
c. & Christian
\end{tabular} & $\begin{array}{l}21 \\
32 \\
7\end{array}$ & $\begin{array}{l}35 \\
53.3 \\
11.7\end{array}$ \\
\hline 4 & \begin{tabular}{ll}
\multicolumn{2}{l}{ Habitat } \\
a. Urban \\
b. Rural
\end{tabular} & $\begin{array}{l}34 \\
26\end{array}$ & $\begin{array}{l}56.7 \\
43.3\end{array}$ \\
\hline 6 & $\begin{array}{l}\text { Type of Family } \\
\text { a. Nuclear } \\
\text { b. Joint }\end{array}$ & $\begin{array}{l}38 \\
22\end{array}$ & $\begin{array}{l}63.3 \\
36.7\end{array}$ \\
\hline 7 & \begin{tabular}{l}
\multicolumn{3}{l}{ Fathers education } \\
a. Primary education \\
b. Secondary education \\
c. $\quad$ Graduation and above
\end{tabular} & $\begin{array}{l}11 \\
31 \\
18\end{array}$ & $\begin{array}{l}18.3 \\
51.7 \\
30\end{array}$ \\
\hline 8 & \begin{tabular}{ll}
\multicolumn{2}{l}{ Mothers education } \\
a. & Primary education \\
b. & Secondary education \\
c. & Graduation and above
\end{tabular} & $\begin{array}{l}7 \\
40 \\
13\end{array}$ & $\begin{array}{l}11.7 \\
66.7 \\
21.6\end{array}$ \\
\hline 11. & $\begin{array}{l}\text { Entertainment facilities } \\
\text { used } \\
\text { a. Television } \\
\text { b. Internet } \\
\text { c. Video games } \\
\text { d. Any other }\end{array}$ & $\begin{array}{l}12 \\
27 \\
3 \\
18\end{array}$ & $\begin{array}{l}20 \\
45 \\
5 \\
30\end{array}$ \\
\hline 12 & \begin{tabular}{ll}
\multicolumn{2}{l}{ Pocket money used on } \\
a. & Entertainment \\
b. & Education \\
c. Junk food \\
d.
\end{tabular} & $\begin{array}{l}4 \\
0 \\
38 \\
18\end{array}$ & $\begin{array}{l}6.7 \\
0 \\
63.3 \\
30 \\
\end{array}$ \\
\hline
\end{tabular}

Table 2: Pre-test and post-test level of happiness of adolescents

\begin{tabular}{|ll|l|l|l|l|}
\hline \multirow{2}{*}{ Level of happiness } & \multicolumn{2}{|l|}{ Pre-test } & \multicolumn{2}{l|}{ Post-test } \\
\cline { 3 - 6 } & f & \% & f & \% \\
\hline$\bullet$ & Greater happiness (80+) & 0 & 0 & 0 & 0 \\
$\bullet$ & High happiness (61-79) & 0 & 0 & 0 & 0 \\
$\bullet$ & Average (31-60) & 0 & 0 & 13 & 21.7 \\
- & Less Happiness (20-30) & 0 & 0 & 13 & 21.7 \\
- & Unhappiness (upto 20) & 60 & 100 & 34 & 56.6 \\
\hline
\end{tabular}

Table 3: Pre-test and Post-test level of psychological well being according to domains among adolescents

\begin{tabular}{|c|c|c|c|c|c|c|c|c|c|c|c|}
\hline \multicolumn{2}{|c|}{ Level of psychological well being } & \multicolumn{2}{|c|}{ Satisfaction } & \multicolumn{2}{|c|}{ Efficiency } & \multicolumn{2}{|c|}{ Sociability } & \multicolumn{2}{|c|}{ Mental health } & \multicolumn{2}{|c|}{ Interpersonal Relation } \\
\hline & & $\mathbf{F}$ & $\%$ & $\mathbf{F}$ & $\%$ & f & $\%$ & f & $\%$ & f & $\%$ \\
\hline \multirow[t]{2}{*}{ Very low (10-12) } & Pre-test & 0 & 0 & 0 & 0 & 0 & 0 & 0 & 0 & 0 & 0 \\
\hline & Post-test & 0 & 0 & 0 & 0 & 0 & 0 & 0 & 0 & 0 & 0 \\
\hline \multirow{2}{*}{$\begin{array}{l}\text { Low } \\
(12-16)\end{array}$} & Pre-test & 16 & 26.7 & 22 & 36.7 & 22 & 36.7 & 34 & 56.7 & 28 & 46.7 \\
\hline & Post-test & 0 & 0 & 0 & 0 & 0 & 0 & 0 & 0 & 0 & 0 \\
\hline \multirow[t]{2}{*}{ Moderate (16-43) } & Pre-test & 44 & 73.3 & 38 & 63.3 & 38 & 63.3 & 26 & 43.3 & 32 & 53.3 \\
\hline & Post-test & 51 & 85 & 47 & 78.3 & 48 & 80 & 46 & 76.7 & 46 & 76.7 \\
\hline \multirow[t]{2}{*}{ High (43-48) } & Pre-test & 0 & 0 & 0 & 0 & 0 & 0 & 0 & 0 & 0 & 0 \\
\hline & Post-test & 9 & 15 & 13 & 21.7 & 12 & 20 & 14 & 23.3 & 14 & 23.3 \\
\hline \multirow[t]{2}{*}{ Very High(48-50) } & Pre-test & 0 & 0 & 0 & 0 & 0 & 0 & 0 & 0 & 0 & 0 \\
\hline & Post-test & 0 & 0 & 0 & 0 & 0 & 0 & 0 & 0 & 0 & 0 \\
\hline
\end{tabular}

Table 3 depicts that in post-test $85 \%$ adolescents had moderate level of psychological well being which was higher than pre-test i.e. $73.3 \%$ adolescents had moderate level of psychological well being
$80 \%$ adolescents had moderate level of psychological well being in post-test and $63.3 \%$ adolescents had moderate level of psychological well being in pre-test which was lower than post-test. 
In efficiency domain $78.3 \%$ adolescents had moderate level of psychological well being in post-test and in pre-test $63.3 \%$ had moderate level of psychological well being which was lower than post-test.

Table 4: Overall Pre-test and Post-test level of psychological well being of adolescents

\begin{tabular}{|l|l|l|l|l|}
\hline \multirow{2}{*}{ Level of psychological well being } & \multicolumn{2}{|l|}{ Pre-test } & \multicolumn{2}{l|}{ Post-test } \\
\cline { 2 - 5 } & F & \% & f & \% \\
\hline Very low (50-58) & 0 & 0 & 0 & 0 \\
\hline Low (58-83) & 30 & 50 & 0 & 0 \\
\hline Moderate (83-217) & 30 & 50 & 45 & 75 \\
\hline High (217-242) & 0 & 0 & 15 & 25 \\
\hline Very High (242-250) & 0 & 0 & 0 & 0 \\
\hline
\end{tabular}

Table 4 depicts that in post-test mean score $75 \%$ of adolescents were having moderate level of psychological well being whereas in pre-test $50 \%$ were having moderate level of psychological well being.
SECTION C: Effectiveness of intervention on level of happiness and psychological well being of adolescents

Table 5: Comparison of pre and post intervention happiness score of adolescents

\begin{tabular}{|c|c|c|c|c|}
\hline Level of Happiness & Mean \pm SD & 't' value & df & 'p' value \\
\hline Pre-test & $50.70 \pm 8.67$ & \multirow[b]{2}{*}{20.30} & \multirow[b]{2}{*}{59} & \multirow[b]{2}{*}{$0.000 * *$} \\
\hline Post-test & $84.83 \pm 12.12$ & & & \\
\hline
\end{tabular}

Table 8 shows that mean post test level of happiness score $(84.83 \pm 12.12)$ is higher that mean pre test score (50.70 \pm 8.67$)$. The computed ' $t$ ' value showed that there is highly significant difference between the pre-test and post-test mean knowledge score $(\mathrm{t}=20.30, \mathrm{p}<0.001)$. Hence research hypothesis is accepted. This indicates that psycho-social nursing intervention was effective in improving the level of happiness among adolescents.

Table 6: Comparison of pre and post intervention psychological well being score of adolescents according to domains

\begin{tabular}{|l|l|l|l|l|l|}
\hline \multirow{2}{*}{ Domains } & Mean \pm SD & 't' value & df & 'p' value \\
\cline { 2 - 4 } & Pre-test & Post-test & & & \\
\hline Satisfaction & $20.03 \pm 4.37$ & $35.06 \pm 5.13$ & 20.59 & 59 & $0.000^{* *}$ \\
\hline Efficiency & $20.80 \pm 6.06$ & $35.26 \pm 5.50$ & 16.26 & 59 & $.0 .001^{*}$ \\
\hline Mociability & $19.70 \pm 5.09$ & $32.86 \pm 7.09$ & 14.24 & 59 & $0.000^{* *}$ \\
\hline Interpersonal helation & $17.93 \pm 4.62$ & $33.90 \pm 9.93$ & 17.20 & 59 & $.0 .000^{* *}$ \\
\hline
\end{tabular}

Note $=* * p$ value $<0.001$ level of significance

Table 6 depicts that the computed ' $t$ ' value showed that there is highly significant difference between the pre-test and post-test psychological well being score in all the domains $(p<0.001)$. Mean post-test score of psychological well being in interpersonal domain $(37.53 \pm 4.83)$ is higher than the mean pre-test psychological well being score $(18.71 \pm 5.22)$.

In efficiency domain mean post-test score of psychological well being $(35.26 \pm 5.50)$ is higher than the mean pretest psychological well being score

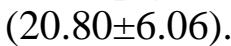

In satisfaction domain the mean score of post-test psychological well being $(35.06 \pm 5.13)$ is higher than the mean score pre-test psychological well being score $(20.03 \pm 4.37)$.

Table 7 shows that mean post test level of psychological well being score $(174.63 \pm 28.01)$ is higher that mean pre test level of psychological well being score (97.18 \pm 22.71$)$. The computed ' $t$ ' value showed that there is highly significant difference between the pre-test and post-test mean knowledge score $(\mathrm{t}=20.61, \mathrm{p}<0.001)$. Hence research hypothesis is accepted. This indicates that psycho-social nursing intervention was effective in improving the level of psychological well being among adolescents.

Table 7: Comparison of pre and post intervention of overall psychological well being of adolescents

\begin{tabular}{|l|l|l|l|l|}
\hline $\begin{array}{l}\text { Psychological Well } \\
\text { being }\end{array}$ & Mean \pm SD & $\begin{array}{l}\text { 't' } \\
\text { value }\end{array}$ & df & $\begin{array}{l}\text { 'p' } \\
\text { value }\end{array}$ \\
\hline Pre-test & $97.18 \pm 22.71$ & 20.61 & 59 & $0.001^{*}$ \\
\hline Post-test & $174.63 \pm 28.01$ & & & \\
\hline
\end{tabular}

Table 8: Co-relation between pre-test and post-test score of happiness and psychological well being of adolescents

\begin{tabular}{|l|l|l|}
\hline \multirow{2}{*}{ Co-relation } & Pre-test & Post-test \\
\cline { 2 - 3 } & Mean \pm SD & Mean \pm SD \\
\hline Happiness & $50.70 \pm 8.67$ & $84.83 \pm 12.12$ \\
\hline Psychological well being & $97.18 \pm 22.71$ & $174.63 \pm 28.01$ \\
\hline 'r' value & 0.044 & 0.132 \\
\hline
\end{tabular}


Table 8 depicts that co-relation between pre-test score of happiness of adolescents (50.70 \pm 8.67$)$ and pre-test score of psychological well being $(97.18 \pm 22.71)$ with the ' $r$ ' value of 0.044 . Post-test score of happiness among adolescents $(84.83 \pm 12.12)$ and post-test score of psychological well being (174.63 \pm 28.01$)$ with the ' $r$ ' value of 0.132 , which is weakly positive in pre-test and post-test.

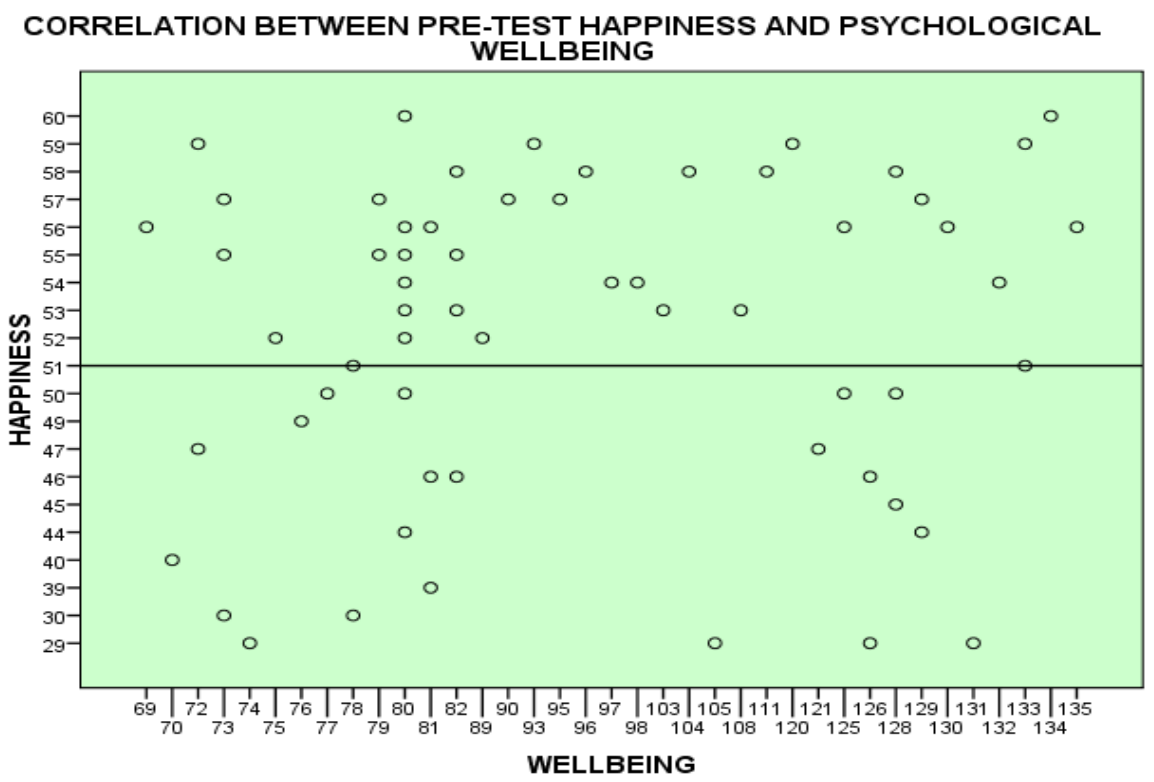

Fig. 7: Correlation between pre-test happiness and psychological well being

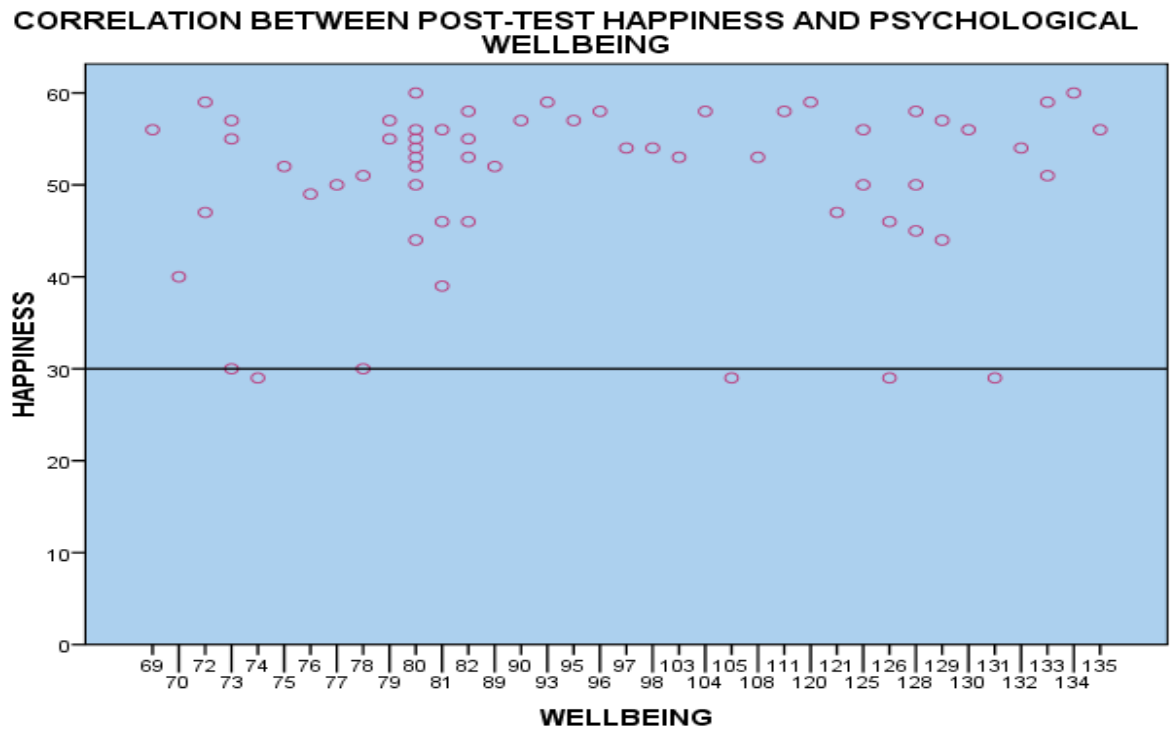

Fig 8: Correlation between post-test happiness and psychological well being

Section D: Association of socio demographic variables with pretest and posttest level of happiness and psychological well being among adolescents.

Data in Table 12 shows the association of post-test level of happiness score with demographic variables which is calculates with the help of ANOVA test. The mean difference of happiness score is higher in primary education in mother (39.87 \pm 4.75$)$ and posttest level of happiness score does not show any statistical significant association with demographic variables. 
Rimplepreet Kaur et.al. A pre-experimental study to assess the effect of psycho-social nursing intervention on happiness and psychological well being among adolescents in selected schools of Amritsar, Punjab.

Table 9: ANOVA of happiness score of adolescents with demographic variables

\begin{tabular}{|c|c|c|c|c|c|c|}
\hline Demographic variable & $\mathbf{n}$ & Mean Gain \pm SD & Df & Sum of Squares & F Value & p value \\
\hline $\begin{array}{l}\text { Age (in Years) } \\
\begin{array}{ll}\text { a. } 14 \mathrm{yrs} \\
\text { b. } 15 \mathrm{yrs} \\
\text { c. } 16 \mathrm{yrs}\end{array} \\
\end{array}$ & $\begin{array}{l}19 \\
20 \\
21\end{array}$ & $\begin{array}{l}31.78 \pm 2.961 \\
34.05 \pm 2.886 \\
36.33 \pm 2.817 \\
\end{array}$ & 2 & 206.15 & 0.619 & $0.542^{\mathrm{NS}}$ \\
\hline $\begin{array}{cl}\text { Religion } & \\
\text { a. } & \text { Sikh } \\
\text { b. } & \text { Hindu } \\
\text { c. } & \text { Christian } \\
\end{array}$ & $\begin{array}{l}21 \\
32 \\
7\end{array}$ & $\begin{array}{l}32.09 \pm 2.81 \\
34.56 \pm 2.28 \\
38.28 \pm 4.87\end{array}$ & 2 & 213.82 & 0.642 & $0.530^{\mathrm{NS}}$ \\
\hline $\begin{aligned} \text { Class } & \\
\text { a. } & 8^{\text {th }} \\
\text { b. } & 9^{\text {th }} \\
\text { c. } & 10^{\text {th }}\end{aligned}$ & $\begin{array}{l}19 \\
20 \\
21\end{array}$ & $\begin{array}{l}31.78 \pm 2.96 \\
34.05 \pm 2.88 \\
36.33 \pm 2.81\end{array}$ & 2 & 206.15 & 0.619 & $0.542^{\mathrm{NS}}$ \\
\hline $\begin{array}{cl}\text { Father's education } \\
\begin{array}{cl}\text { a. } & \text { Primary education } \\
\text { b. } & \text { Secondary education } \\
\text { c. } & \text { Graduation and above }\end{array}\end{array}$ & $\begin{array}{l}11 \\
31 \\
18\end{array}$ & $\begin{array}{l}31.81 \pm 3.89 \\
35.81 \pm 2.31 \\
32.55 \pm 3.04\end{array}$ & 2 & 197.36 & 0.592 & $0.557^{\mathrm{NS}}$ \\
\hline $\begin{array}{cl}\text { Mother's education } \\
\text { a. } & \text { Primary education } \\
\text { b. } & \text { Secondary education } \\
\text { c. } & \text { Graduation and above }\end{array}$ & $\begin{array}{l}7 \\
40 \\
13\end{array}$ & $\begin{array}{l}39.87 \pm 4.75 \\
31.77 \pm 1.99 \\
38.30 \pm 3.49\end{array}$ & 2 & 678.33 & 2.14 & $0.127^{\mathrm{NS}}$ \\
\hline $\begin{array}{cl}\text { Entertainment facilities used } \\
\begin{array}{cl}\text { a. } & \text { Television } \\
\text { b. } & \text { Internet } \\
\text { c. } & \text { Video games } \\
\text { d. } & \text { Any other }\end{array}\end{array}$ & $\begin{array}{l}12 \\
27 \\
3 \\
18\end{array}$ & $\begin{array}{l}29.83 \pm 3.73 \\
36.00 \pm 2.49 \\
35.00 \pm 7.47 \\
34.05 \pm 3.05\end{array}$ & 3 & 318.32 & 0.63 & $0.597^{\mathrm{NS}}$ \\
\hline $\begin{array}{cl}\text { Pocket money used on } \\
\text { a. } & \text { Entertainment } \\
\text { b. } & \text { Junk food } \\
\text { c. } & \text { Any other }\end{array}$ & $\begin{array}{l}4 \\
0 \\
38 \\
18\end{array}$ & $\begin{array}{l}40.00 \pm 6.46 \\
0 \\
33.21 \pm 34.77 \\
34.77 \pm 3.04\end{array}$ & 2 & 177.50 & 0.531 & $0.591^{\mathrm{NS}}$ \\
\hline
\end{tabular}

TABLE 10: Comparison of happiness score of adolescents with demographic variables

\begin{tabular}{|c|c|c|c|c|c|}
\hline Demographic variables & $\mathbf{N}$ & Mean Gain \pm SD & df & t value & p value \\
\hline $\begin{aligned} & \text { Gender } \\
& \text { a. } \text { Male } \\
& \text { b. } \text { Female } \\
&\end{aligned}$ & $\begin{array}{l}30 \\
30 \\
\end{array}$ & $\begin{array}{l}35.03 \pm 12.71 \\
33.23 \pm 13.08 \\
\end{array}$ & 58 & 0.540 & $0.591^{\mathrm{NS}}$ \\
\hline $\begin{array}{cl}\text { Habitat } & \\
\text { a. } & \text { Urban } \\
\text { b. } & \text { Rural } \\
\end{array}$ & $\begin{array}{l}34 \\
26 \\
\end{array}$ & $\begin{array}{l}35.03 \pm 12.71 \\
33.23 \pm 13.08\end{array}$ & 58 & -0.94 & $0.68^{\mathrm{NS}}$ \\
\hline $\begin{array}{cl}\text { Type of family } \\
\begin{aligned} & \text { a. } \text { Nuclear } \\
& \text { b. } \text { Joint } \\
&\end{aligned}\end{array}$ & $\begin{array}{l}38 \\
22 \\
\end{array}$ & $\begin{array}{l}34.71 \pm 13.38 \\
33.14 \pm 12.02 \\
\end{array}$ & 58 & 0.45 & $0.314^{\mathrm{NS}}$ \\
\hline $\begin{array}{cc}\text { Father's occupation } \\
\text { a. } & \text { Unemployed } \\
\text { b. } & \text { Employed } \\
\end{array}$ & $\begin{array}{l}0 \\
60 \\
\end{array}$ & $\begin{array}{l}0 \\
34.13 \pm 12.84\end{array}$ & - & - & - \\
\hline $\begin{array}{cl}\text { Mother's occupation } \\
\text { a. } & \text { Unemployed } \\
\text { b. } & \text { Employed } \\
\end{array}$ & $\begin{array}{l}27 \\
33 \\
\end{array}$ & $\begin{array}{l}34.41 \pm 12.15 \\
33.91 \pm 13.531 \\
\end{array}$ & 58 & 0.88 & $0.47^{\mathrm{NS}}$ \\
\hline
\end{tabular}

Note $=$ NS $=$ Non Significant at $p$ value $>0.005, d f=$ degree of freedom

Table 11: ANOVA of psychological well being score of adolescents with demographic variables

\begin{tabular}{|c|c|c|c|c|c|c|}
\hline Demographic variable & $\mathbf{n}$ & Mean Gain \pm SD & Df & Sum of Squares & F Value & p value \\
\hline $\begin{array}{c}\text { Age (in Years) } \\
\begin{array}{cc}\text { a. } & 14 \mathrm{yrs} \\
\text { b. } & 15 \mathrm{yrs} \\
\text { c. } & 16 \mathrm{yrs}\end{array}\end{array}$ & $\begin{array}{l}19 \\
20 \\
21\end{array}$ & $\begin{array}{c}73.47 \pm 6.69 \\
87.450 \pm 6.52 \\
71.52 \pm 6.36\end{array}$ & 2 & 30.37 .92 & 1.78 & $0.17^{\mathrm{NS}}$ \\
\hline $\begin{array}{cl}\text { Religion } & \\
\text { a. } & \text { Sikh } \\
\text { b. } & \text { Hindu } \\
\text { c. } & \text { Christian } \\
\end{array}$ & $\begin{array}{l}21 \\
32 \\
7\end{array}$ & $\begin{array}{l}77.52 \pm 6.65 \\
77.12 \pm 5.31 \\
78.71 \pm 11.36\end{array}$ & 2 & 14.68 & 0.008 & $0.99^{\mathrm{NS}}$ \\
\hline $\begin{aligned} \text { Class } & \\
\text { a. } & 8^{\text {th }} \\
\text { b. } & 9^{\text {th }} \\
\text { c. } & 10^{\text {th }}\end{aligned}$ & $\begin{array}{l}19 \\
20 \\
21\end{array}$ & $\begin{array}{l}73.47 \pm 6.69 \\
87.45 \pm 6.52 \\
71.52 \pm 6.36\end{array}$ & 2 & 3037.92 & 1.78 & $0.17^{\mathrm{NS}}$ \\
\hline $\begin{array}{l}\text { Fathers education } \\
\begin{array}{cl}\text { a. } & \text { Primary education } \\
\text { b. } & \text { Secondary education } \\
\text { c. } & \text { Graduation and above }\end{array}\end{array}$ & $\begin{array}{l}11 \\
31 \\
18\end{array}$ & $\begin{array}{l}75.63 \pm 9.06 \\
77.63 \pm 5.39 \\
78.38 \pm 7.08\end{array}$ & 2 & 52.34 & 0.29 & $0.97^{\mathrm{NS}}$ \\
\hline
\end{tabular}


Rimplepreet Kaur et.al. A pre-experimental study to assess the effect of psycho-social nursing intervention on happiness and psychological well being among adolescents in selected schools of Amritsar, Punjab.

\begin{tabular}{|c|c|c|c|c|c|c|}
\hline \multicolumn{7}{|c|}{ Table 11: Continued... } \\
\hline Mothers education & \multirow{4}{*}{$\begin{array}{l}7 \\
40 \\
13\end{array}$} & \multirow{4}{*}{$\begin{array}{l}75.28 \pm 11.33 \\
78.90 \pm 4.74 \\
74.15 \pm 8.31\end{array}$} & \multirow{4}{*}{2} & \multirow{4}{*}{258.12} & \multirow{4}{*}{0.143} & \multirow{4}{*}{$0.86^{\mathrm{NS}}$} \\
\hline a. Primary education & & & & & & \\
\hline b. Secondary education & & & & & & \\
\hline c. Graduation and above & & & & & & \\
\hline Entertainment facilities used & & & & & & \\
\hline a. Television & 12 & $79.08 \pm 8.74$ & & 155.85 & 0.57 & $0.98^{\mathrm{NS}}$ \\
\hline Internet & 27 & $75.70 \pm 5.82$ & 3 & & & \\
\hline Video games & 3 & $80.00 \pm 17.48$ & & & & \\
\hline d. Any other & 18 & $78.55 \pm 7.13$ & & & & \\
\hline Pocket money used on & & & & & & \\
\hline a. Entertainment & 4 & $71.25 \pm 14.96$ & & 460.18 & 0.25 & $0.77^{\mathrm{NS}}$ \\
\hline b. Education & 0 & & 2 & & & \\
\hline c. Junk food & 38 & $79.47 \pm 4.85$ & & & & \\
\hline d. Any other & 18 & $74.55 \pm 7.05$ & & & & \\
\hline
\end{tabular}

Table 13 depicts the comparison of happiness score with demographic variables in which male from gender and urban from habitat has higher mean gain $(35.03 \pm 12.71)$ posttest level of happiness score does not show any statistical significant association with demographic variables.
Table 14 depicts the comparison of psychological well being score with demographic variables in which internet from entertainment has higher mean gain $(80.00 \pm 17.48)$ and posttest level of psychological well being score does not show any statistical significant association with demographic variables

\begin{tabular}{l} 
TABLE 12: Comparison of happiness score of adolescents with demographic variables \\
\begin{tabular}{|rl|l|l|l|l|l|}
\hline Demographic variables & N & Mean Gain \pm SD & Df & t value & p value \\
\hline $\begin{array}{r}\text { Gender } \\
\text { c. Male }\end{array}$ & 30 & $77.43 \pm 24.88$ & 58 & -0.004 & $0.05^{\mathrm{NS}}$ \\
d. Female & 30 & $77.47 \pm 34.01$ & & & \\
\hline $\begin{array}{r}\text { Habitat } \\
\text { c. Urban }\end{array}$ & 34 & $70.56 \pm 30.36$ & 58 & -2.12 & $0.12^{\mathrm{NS}}$ \\
d. Rural & 26 & $86.36 \pm 26.36$ & & & \\
\hline $\begin{array}{c}\text { Type of family } \\
\text { c. Nuclear } \\
\text { d. Joint }\end{array}$ & 38 & $74.61 \pm 29.62$ & 58 & -0.98 & $0.99^{\mathrm{NS}}$ \\
\hline $\begin{array}{c}\text { Father's occupation } \\
\text { c. Unemployed } \\
\text { d. Employed }\end{array}$ & 0 & 0 & $82.36 \pm 29.44$ & & & \\
\hline $\begin{array}{c}\text { Mother's occupation } \\
\text { c. Housewife }\end{array}$ & 60 & $77.45 \pm 29.55$ & 58 & - & - \\
d. Employed & 27 & $76.74 \pm 28.51$ & 58 & -1.67 & $0.76^{\mathrm{NS}}$ \\
\hline
\end{tabular} \\
Note $=$ NS = Non Significant at $p$ value >0.005, df= degree of freedom \\
\hline
\end{tabular}

Table 15 depicts the comparison of psychological well being score with demographic variables in which rural from habitat has higher mean gain $(86.36 \pm 26.36)$ and posttest level of psychological well being score does not show any statistical significant association with demographic variables.

\section{CONCLUSION}

It was concluded from present study that the psycho-social nursing intervention is effective in improving the level of happiness and psychological well being among adolescents and there is great need to in-corporate complementary therapies in enhancing happiness and psychological well being of adolescents in schools.

Acknowledgement: None

Conflict of Interest: None

Source of Funding: None

Ethical Approval: Approved

\section{REFERENCES}

1. Omobuwa O, Alebiosu CO, Olajide FO, Adepime WO. Journal of South Africa Family Practice. 2014;56(2):246-250. Available from; 
Rimplepreet Kaur et.al. A pre-experimental study to assess the effect of psycho-social nursing intervention on happiness and psychological well being among adolescents in selected schools of Amritsar, Punjab.

https://www.tandfonline.com/doi/full/10.10 80/20786190.2014.953891

2. Orphan H. Correlates of well-being among Canadians with mood and/or anxiety disorders, health promotion and chronic disease prevention in Canada available from:

https://www.ncbi.nlm.nih.gov/pmc/articles/ PMC5387797/

3. Boiler L, Haverman M, Westerhof $\mathrm{J}$ G, Riper H, Smit F, Bohlmeijer E. Positive psychology interventions: a meta-analysis of randomized controlled studies. BMC Public Health. 2013;13:119.

4. Levy S. Introduction to Problems in Adolescents. Available from:

5. https://www.msdmanuals.com/enin/home/children-s-health-issues/problemsin-adolescents/introduction-to-problems-inadolescents

6. Wai C, Cherry A. A Positive Education Program to Promote Well Being in school a case study from a Hong Kong school.

7. HutaV,(2015). An overview of hedonic and eudaimonic well-being concepts. Available from:

https://www.researchgate.net/publication/29 2134516_An_overview_of_hedonic_and_eu daimonic_well-being_concepts
8. Baltes, P, Freund, A. Human strengths as the orchestration of wisdom and selective optimization with compensation. In L. G. Aspinwall \& U. M. Staudinger (Eds.), A psychology of human strengths: Fundamental questions and future directions for positive psychology, Washington, D.C. American Psychological Association. 2003.

9. Esposito Linda exercises from positive psychology to boost happiness. Available from: https://tinybuddha.com/blog/6exercises-positive-psychology-boosthappiness/

10. Baltes P, Freund A. Human strengths as the orchestration of wisdom and selective optimization with compensation. A psychology of human strengths: Fundamental questions and future directions for positive psychology, Washington, D.C.: American Psychological Association:2003

How to cite this article: Kaur R, Kaur S, Kaur G. A pre-experimental study to assess the effect of psycho-social nursing intervention on happiness and psychological well being among adolescents in selected schools of Amritsar, Punjab. Int J Health Sci Res. 2021; 11(4):144152. DOI: https://doi.org/10.52403/ijhsr. 20210419 